\title{
The membrane-spanning domain of gp41 plays a critical role in intracellular trafficking of the HIV envelope protein
}

Kosuke Miyauchi ${ }^{1}$, A Rachael Curran ${ }^{2}$, Yufei Long ${ }^{3}$, Naoyuki Kondo ${ }^{3,5,6}$, Aikichi Iwamoto ${ }^{4}$, Donald M Engelman ${ }^{2}$, Zene Matsuda ${ }^{3,5^{*}}$

\begin{abstract}
Background: The sequences of membrane-spanning domains (MSDs) on the gp41 subunit are highly conserved among many isolates of HIV-1. The GXXXG motif, a potential helix-helix interaction motif, and an arginine residue (rare in hydrophobic MSDs) are especially well conserved. These two conserved elements are expected to locate on the opposite sides of the MSD, if the MSD takes a $\alpha$-helical secondary structure. A scanning alanine-insertion mutagenesis was performed to elucidate the structure-function relationship of gp41 MSD.

Results: A circular dichroism analysis of a synthetic gp41 MSD peptide determined that the secondary structure of the gp41 MSD was $\alpha$-helical. We then performed a scanning alanine-insertion mutagenesis of the entire gp41 MSD, progressively shifting the relative positions of MSD segments around the helix axis. Altering the position of Gly694, the last residue of the GXXXG motif, relative to Arg696 (the number indicates the position of the amino acid residues in HXB2 Env) around the axis resulted in defective fusion. These mutants showed impaired processing of the gp160 precursor into gp120 and gp41. Furthermore, these Env mutants manifested inefficient intracellular transport in the endoplasmic reticulum and Golgi regions. Indeed, a transplantation of the gp41 MSD portion into the transmembrane domain of another membrane protein, Tac, altered its intracellular distribution. Our data suggest that the intact MSD $\alpha$-helix is critical in the intracellular trafficking of HIV-1 Env.
\end{abstract}

Conclusions: The relative position between the highly conserved GXXXG motif and an arginine residue around the gp41 MSD $\alpha$-helix is critical for intracellular trafficking of HIV-1 Env. The gp41 MSD region not only modulates membrane fusion but also controls biosynthesis of HIV-1 Env.

\section{Background}

HIV-1, the retrovirus responsible for the current worldwide AIDS pandemic, is an enveloped virus. The envelope protein (Env) of HIV-1 is essential for determining host range and for inducing the membrane fusion that allows the virus to enter the host cell. The former and latter functions are mediated by the SU (gp120) and the TM (gp41) subunits of the envelope protein, respectively [1-3]. The SU and TM are generated from a precursor (gp160) by cellular proteases that recognize a basic amino acid sequence between gp120 and gp41 [4-6].

\footnotetext{
* Correspondence: zmatsuda@ims.u-tokyo.ac.jp

${ }^{3}$ China-Japan Joint Laboratory of Structural Virology and Immunology, Institute of Biophysics, Chinese Academy of Sciences, 15 Datun Road, Beijing, 100101 PR China

Full list of author information is available at the end of the article
}

This proteolytic processing is essential to generate fusion-competent HIV-1 Env and is believed to take place in an early Golgi region $[7,8]$.

HIV-1 Env is anchored across lipid bilayers via its highly conserved membrane-spanning domain (MSD) [9]. Although the possibility of a transient alteration of the membrane topology exists [10,11], HIV-1 Env is widely believed to be a type I membrane protein with a single $\alpha$-helical MSD in the steady state [12]. Two different models exist within the single MSD model of HIV-1 Env. In an initial model, the MSD is supposed to be 23 amino acid residues long, ranging from Lys683 to Val704 in the HXB2 sequence, and has a highly conserved hydrophilic arginine residue in the midst of its hydrophobic amino acid sequence [13]. In an alternative model, MSD is shorter; and the arginine residue in the 
lipid bilayer is expected to interact with the polar head of the lipid molecule $[14,15]$.

The primary structure of the MSD of HIV-1 Env also has a GXXXG motif, a motif often found at the helixhelix interface of transmembrane $\alpha$-helices [16]; it exists upstream of the arginine residue. If an ordinary $\alpha$-helix structure is assumed for the MSD, the GXXXG motif and arginine residue are positioned on opposite sides of the gp41 MSD $\alpha$-helix.

In vitro studies of the gp41 MSD showed a high tolerance for mutations. For example, the above mentioned conserved arginine residue [17] and the GXXXG motif can accommodate point mutations [18]. Even several heterologous MSDs can replace the entire gp41 MSD without deteriorating effects $[17,19]$. These findings led to the notion that the specific amino acid sequence in the gp41 MSD has no significant biological role within the limits of the assays used. This is a curious notion since the sequence is quite conserved in nature, despite the virus being subject to very strong sequence diversification from errors in reverse transcription.

In fact, other studies have suggested that the specific sequence of the gp41 MSD plays a role in the function of gp41 $[20,21]$. We have shown that replacing the gp41 MSD with MSDs derived from glycophorin A or VSV-G, each containing the GXXXG motif, severely decreases the fusion activity of HIV-1 Env [18,22]. Furthermore, simultaneous substitution of all three glycine residues, within the GXXXG motif with leucine residues, also negatively affected the function of the HIV-1 Env [23]. Shang et al. recently showed the importance of the GXXXG region using a unique genetic approach [24]. These studies clearly suggested the presence of important information encoded in the sequence of MSD. However, the nature of the code is still not evident.

To further elucidate the structure-function relationship of the gp41 MSD, we analyzed a circular dichroism $(\mathrm{CD})$ profile of the synthetic peptide corresponding to the MSD and obtained the profile expected for $\alpha$-helical secondary structure. Next, we used the envelope gene of HXB2 [25] to create a series of alanine insertion mutants of the entire predicted MSD. We found that alteration of the relationship between Gly694 and Arg696 (the number indicates the position of the amino acid residues in HXB2 Env) around the axis of the MSD $\alpha$-helix resulted in fusion incompetent Env. These mutant Envs also showed defects in proteolytic processing and intracellular transport in the endoplasmic reticulum (ER) and Golgi regions. We further showed that the intracellular transport of HIV-1 Env is regulated by the MSD region, through experiments that transplanted the gp41 MSD into another membrane protein, Tac.
This transplantation led to an alteration of the intracellular distribution of Tac, similar to that of HIV-1 Env.

\section{Results}

Circular dichroism analysis of the synthetic MSD peptide in lipid shows $\alpha$-helical secondary structure

The primary structure of the gp41 MSD is highly conserved, and its secondary structure has been predicted to be an $\alpha$-helix based on computational algorithms [26]. However, there are no physical data to support this expectation. We synthesized a peptide corresponding to a consensus HIV-1 clade B structure of the gp41 MSD and determined its CD spectrum in lipid bilayers. The $\mathrm{CD}$ profile, shown in Figure 1, has negative maxima near $208 \mathrm{~nm}$ and $222 \mathrm{~nm}$, indicating the presence of an $\alpha$-helical structure. Although the gp41 MSD of HIV-1 contains three glycine residues, thought to be helixbreaking residues in soluble proteins, the dominant structure indicated by our CD data was an $\alpha$-helix. Many glycines are found in transmembrane helices. Addition of lysine residues at both ends was necessary to allow us to purify the extremely hydrophobic MSD peptide. We cannot completely exclude the possibility that these lysine residues at the termini, especially at the C-terminus, may stabilize the $\alpha$-helical structure.

\section{Scanning alanine-insertion mutagenesis identified the region of gp41 MSD critical for membrane fusion}

To identify the region of the gp41 MSD $\alpha$-helix critical for its function, we generated a set of alanine-insertion mutants covering the entire predicted MSD by using the HXB2 envelope gene. The alanine residue was chosen because it can be well accommodated in an $\alpha$-helix $[27,28]$. Since previous data suggest the involvement of the gp41 MSD in membrane fusion [18,23,24,29], membrane fusion activity was determined for the mutants. The primary structures of these mutants are shown in Figure 2. Nomenclature is based on the positions of the inserted alanine residues in HIV-1 Env. Therefore, 684 + A mutant indicates that the inserted alanine residue corresponds to the 684th residue of the envelope protein. The mutant envelope gene was cloned into the envelope expression vector, and the fusion activity of each mutant was determined by the T7 RNA polymerase transfer assay as described previously [18]. The result is shown in Figure 3A. Among the twenty-two mutants we generated, three showed a prominent decrease in the fusion activity. These three are $694+\mathrm{A}$, $695+\mathrm{A}$, and $696+\mathrm{A}$; their relative fusion activities when compared with the wild type (WT) were $37.5 \%, 14.0 \%$ and $15.5 \%$, respectively. Mutants $695+\mathrm{A}$ and $696+\mathrm{A}$ showed more severe defects than $694+\mathrm{A}$. Thus the corresponding region from 694 to 696 , the $G^{694} L^{696}$ region, was shown to be critical for fusion activity. 


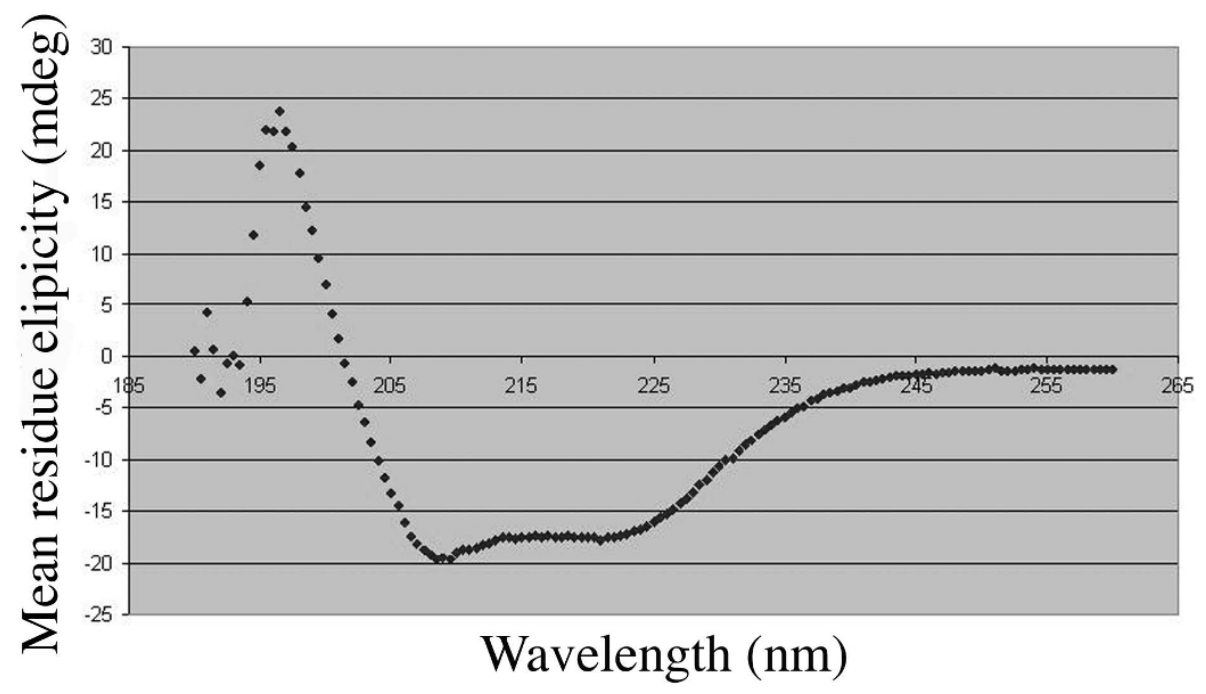

Figure 1 The circular dichroism (CD) profile of the synthetic MSD peptide. The synthetic peptide was dissolved in 15 mM DPC ( $\mathrm{n}$-dodecyl pyridinium chloride), $20 \mathrm{mM} \mathrm{NaPi}, 150 \mathrm{mM} \mathrm{NaCl}$. The spectrum information was collected as described in the materials and methods section. The diagram shown is the average of eight spectra.

The alteration of the phase of the GLR region in MSD was critical to membrane fusion

The insertion of an alanine residue affects both the length and the phase of the $\alpha$-helix. However, we expected that the local phase might have a more important role than the length of the MSD because all the insertion mutants generated are expected to have the same length of the MSD. To verify this, we inserted one, two, three, and four alanine residues between residues 694 and 695 (Figure 2 bottom, 695+2A, 3A, and $4 \mathrm{~A})$ and examined the fusion activities of each resulting mutant. The result is shown in Figure 3B. The insertion of two residues caused a further decrease in the fusion activity compared to the single insertion (compare 695 $+\mathrm{A}$ and $695+2 \mathrm{~A}$ ). However, the fusion activity slightly recovered with the insertion of three residues, and almost fully recovered by the insertion of four alanine residues. It seems that there is a correlation between the recovery of the phase of gp41 MSD $\alpha$-helix and recovery of membrane fusion activity. The observed defect in fusion activity was not due to the increase in length but instead to the local shift of the gp41 MSD $\alpha$-helix.

To further identify residues critical for determining the phase in the GLR region, we generated $696+2 \mathrm{~A}$ and $695 / 696+2 \mathrm{~A}$ (a combination of $695+2 \mathrm{~A}$ and $696+2 \mathrm{~A}$, Figure 2 bottom) and then compared the fusion activity together with $695+2 \mathrm{~A}$. Both $695+2 \mathrm{~A}$ and $696+2 \mathrm{~A}$ showed severe defects in membrane fusion (Figure $3 \mathrm{C}$ ). Interestingly, the combination of these two $(695 / 696$ $+2 \mathrm{~A}$ ) recovered fusion activity. The phase commonly altered in the fusion defective mutants, 695+2A and 696 $+2 \mathrm{~A}$, but corrected in the fusion competent $695 / 696+2 \mathrm{~A}$ mutants was found to be between Gly694 and Arg696. Thus the relationship between Gly694 and Arg696 seems to be an important factor for the membrane fusion activity.

\section{Analysis of the protein profile of the fusion-defective mutants reveals impaired processing of gp160 into gp120 and gp41}

We analyzed the protein profiles of these mutant Envs by immunoblotting, using anti-gp120 and anti-gp41 antibodies (Figure 4). All mutant Envs were expressed at comparable levels (Figure 4A); however, the fusiondefective mutants had impaired processing of gp160, namely more gp160 than gp120; and accordingly less gp41 (see 694+A, 695+A, and 696+A) was observed. This tendency was more prominent for $695+2 \mathrm{~A}$ and 696 $+2 \mathrm{~A}$, each of which showed severe defects in fusion. A similar correlation between impaired processing of Env and defective membrane fusion was observed in the multiple alanine insertion mutants that showed defective fusion (Figure 3C and 4B). Because the generation of processed gp41 is a prerequisite for fusion competency, this protein profile for inefficient gp160 processing is consistent with the observed fusion defect. Our data showed that the alteration in the $\alpha$-helical phase in the localized region within gp41 MSD affected processing of gp160 into gp120 and gp41. It was also shown that these mutants were fusion incompetent. A possibility is that the mutations induced allosteric structural changes of the cleavage site so that the mutant Env was no longer processed properly by Furin or Furin-like proteases. However, this idea was not supported by the 
WT: yikLFIMIVGGLVGLRIVFAVLSIVnrV

684+A: yi kALFIMIVGGLVGLRIVFAVLSIVnrV 685+A: yikLAFINIVGGLVGLRIVFAVLSIVnrV 686+A : yikLFAIMIVGGLVGLRIVFAVLSIVnrV 687+A : yikLFIAMIVGGLVGLRIVFAVLSIVnrV 688+A : yikLFIMAIVGGLVGLRIVFAVLSIVnrV 689+A : yikLFIMIAVGGLVGLRIVFAVLSIVnrV 690+A: yi KLFIMIVAGGLVGLRIVFAVLSIVnrV 691+A : yi kLFIMIVGAGLVGLRIVFAVLSIVnrV 692+A : yikLFIMIVGGALVGLRIVFAVLSIVnrV 693+A: yikLFIMIVGGLAVGLRIVFAVLSIVnrV 694+A: yikLFIMIVGGLVAGLRIVFAVLSIVnrV 695+A : yikLFIMIVGGLVGALRIVFAVLSIVnrV 696+A : YikLFIMIVGGLVGLARIVFAVLSIVnrV 697+A : yikLFIMIVGGLVGLRA_IVFAVLSIVnrV 698+A : yikLFIMIVGGLVGLRIAVFAVLSIVnrV 699+A: yikLFIMIVGGLVGLRIVAFAVLSIVnrV 700+A : yikLFIMIVGGLVGLRIVFA_AVLSIVnrV 702+A: yikLFIMIVGGLVGLRIVFAVALSIVnrV 703+A : yikLFIMIVGGLVGLRIVFAVLASIVnrV 704+A : yikLFIMIVGGLVGLRIVFAVLSÄIVnrV 705+A: yikLFIMIVGGLVGLRIVFAVLSIAQVnrV 706+A : yikLFIMIVGGLVGLRIVFAVLSIVAnrV

695+2A: yikLFINTVGGLVGAALRIVFAVLSIVnrV 695+3A: yikLFIMTVGGLVGAAALRIVFAVLSIVnrv 695+4A: yikLFIMTVGGLVGAAAALRIVFAVLSTVnrv

696+2A: yikLFIMIVGGLVGLAARIVFAVLSIVnrV 695/696+2A: yikLFIMTVGGLVGAALAARIVFAVLSIVnrV

Figure 2 Amino acid sequences of the MSD of the wild type (WT) and Ala-insertion mutants used in this study. The predicted MSD portion is indicated in capital letters. The inserted alanine residue is underlined.

observation that mutant gp160, purified from COS-7 cells, is cleaved into the gp120 and gp41 subunits by commercially available Furin in vitro (Additional file 1). We also analyzed the trimerization of Env mutants.
The trimer of $695+2 \mathrm{~A}$ Env was detected (Additional file 2) However, the presence of less drastic yet critical structural alterations by the mutation cannot be ruled out completely.

\section{Alanine insertion in the gp41 MSD can alter the intracellular distribution of Env}

Since processing of gp160 takes place in the Golgi $[7,8]$, we hypothesized that the defect in the processing was derived from the defect in the intracellular trafficking of mutant Env in the endoplasmic reticulum and Golgi regions. To test this possibility, we examined the distribution of mutant Env in the cells. We attached a FLAG tag at the C-terminus of gp41, providing a linear epitope that can be recognized by monoclonal antibody, M2. An ttachment of the FLAG tag did not alter the defect in processing present in alanine insertion mutants (data not shown). The envelope proteins expressed in COS-7 cells were visualized by immunofluorecent assay using the anti-FLAG monoclonal antibody. We observed that fine, mesh-like fluorescent signals distributing within the transfected cells were more prominent for the mutant $695+2 \mathrm{~A}$ than the WT (Figure 5). The intensity of fluorescence derived from Env at the Golgi area was notably weaker for $695+2 \mathrm{~A}$ than for the WT. These data suggested that mutant Env was defective for transport from ER to Golgi. The level of Env expressed on the cell surface, analyzed by FACS, is consistent with this observation because it is lower for the mutant than for the WT (Figure 6).

To further verify the transport defect biochemically, we analyzed the pattern of modification of sugar moieties in the WT and mutant Env. The results are shown in Figure 7. When treated with endoglycosidase $\mathrm{H}$ (Endo $\mathrm{H}$ ), the WT exhibited an Endo H-resistant fraction of gp160 whereas almost no Endo H-resistant gp160 was detected in the $695+2 \mathrm{~A}$ mutant. This finding indicated that sugar moieties attached to the mutant envelope protein remained as high-mannose types. However, both the WT and mutant envelope proteins generated bands that migrated similarly after treatment with Peptide: N-Glycosidase F (PNGase F), which cleaves between the innermost GlcNAc, and asparagine residues, where sugar moieties are attached. These data further confirmed the defect of the mutant envelope protein in transport, probably in ER-Golgi regions.

\section{The transfer of the gp41 MSD into a foreign membrane} protein alters the intracellular distribution of chimeric proteins

We are interested in determining whether the MSD region alone is sufficient to induce the observed transport defect in the context of other membrane proteins. To test this possibility we have replaced the MSD of 


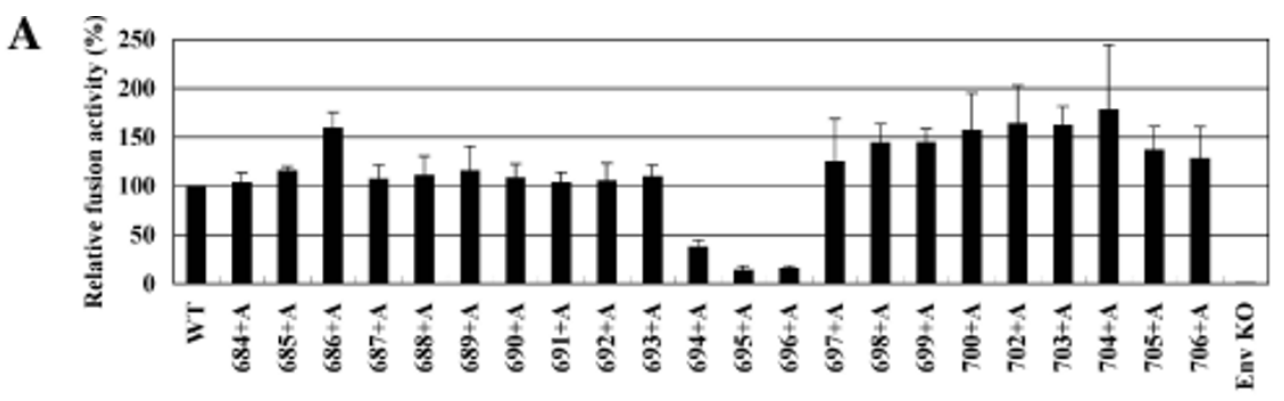

B

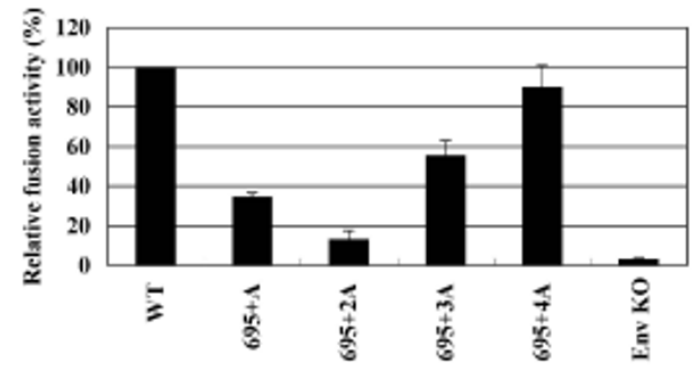

C

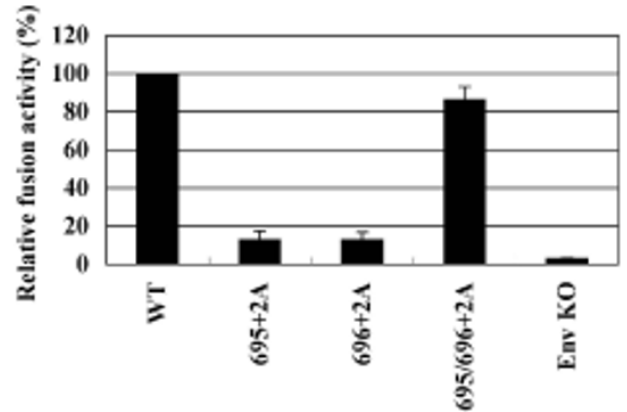

Figure 3 The fusion activity of Ala-insertion mutants in the cell-cell fusion assay. COS-7 cells transfected with the T7 RNA polymerase expression vector and the Env expression vector were co-cultured with 293CD4 cells transfected with a plasmid containing T7 promoter-driven renilla luciferase reporter. After a 24-hr co-culture, the renilla luciferase reporter activity was measured and normalized to the firefly activities as described previously [18]. The normalized renilla luciferase activities for (A) single Ala-inserted mutant of Env, (B) the mutant Env with multiple Ala insertion, $(C)$ mutant Env with two alanine residues inserted at positions 695 and 696 are shown. Data are the average of three independent experiments. The error bar indicates a standard error.

Tac, the $\alpha$-chain of the Interleukin- 2 receptor, with the MSD of the wild type (Tac-gp41WT) or $695+2 \mathrm{~A}$ mutant (Tac-gp41+2A) of gp41, and determined the intracellular distribution of the engineered Tac proteins. We included the intact Tac as a reference (Tac-WT). The results are shown in Figure 8. The signals of intact Tac proteins distributed both in the cytoplasm and plasma membrane areas. They show a fine mesh like appearance in the cytoplasm and are well overlapped with the signals of the ER markers. The intact Tac proteins also showed prominent signals at the rim of the cells suggesting efficient transport to the plasma membrane (Figure $8 \mathrm{~A}$ and $8 \mathrm{D}$ ). There was no overlap of signals for intact Tac and Golgi markers (Figure $8 \mathrm{G}$ ). When the MSD of intact Tac proteins was replaced with that of gp41 (wild type in Figure 8B and $8 \mathrm{E} ; 695+2 \mathrm{~A}$ in $\mathrm{C}$ and $\mathrm{F}$ ), the signals corresponding to the plasma membrane areas became weaker than those of intact Tac (Figure 8, compare A with B and C; D versus $E$ and F). The majority of the signals was observed in the cytoplasm, and the signals were co-localized with ER markers (Figure $8 \mathrm{~B}$ and $8 \mathrm{C}$ ). There are some signals of Tac-gp41 chimera in Golgi areas (Figure $8 \mathrm{H}$ and 8I). Different from the context of HIV-1 envelope proteins (Figure 5E and 5F), we did not detect a discernable difference in the distribution between the wild type gp41 MSD (Figure 8 Tac-gp41WT) and 695+2A gp41 MSD (Tac-
gp41+2A) in the Golgi areas (Figure $8 \mathrm{H}$ and $8 \mathrm{I}$ ). It appeared that the introduction of the gp41 MSD made chimeric Tac distribute in the cytoplasmic region, mainly ER regions, but the difference between the wild type gp41 and 695+2A mutant became less prominent in the context of Tac than in the context of the HIV-1 Env.

\section{Discussion}

Although the gp41 MSD has three glycine residues, our $\mathrm{CD}$ analysis suggested the presence of the $\alpha$-helical structure in gp41 MSD (Figure 1). This may not be a surprise, since glycines are abundant in transmembrane helices and glycines are viewed as helix breakers in soluble proteins. A recent molecular dynamics study also supports a helical conformation [30]. Furthermore, the replacement of all three glycine residues with alanine residues, highly $\alpha$-helix-forming residues $[27,28]$, did not affect the fusion activity of gp41 [18]. Thus gp41 MSD is presumably functional with an $\alpha$-helical structure. These data, however, do not rule out the possibility of the reported transient alteration of the secondary structure of the gp41 MSD during membrane fusion [11].

Our scanning alanine insertion mutagenesis identified the topological relationship between Gly694 and Arg696 around the MSD $\alpha$-helix as a critical determinant for the proper processing (Figure 4) and intracellular 

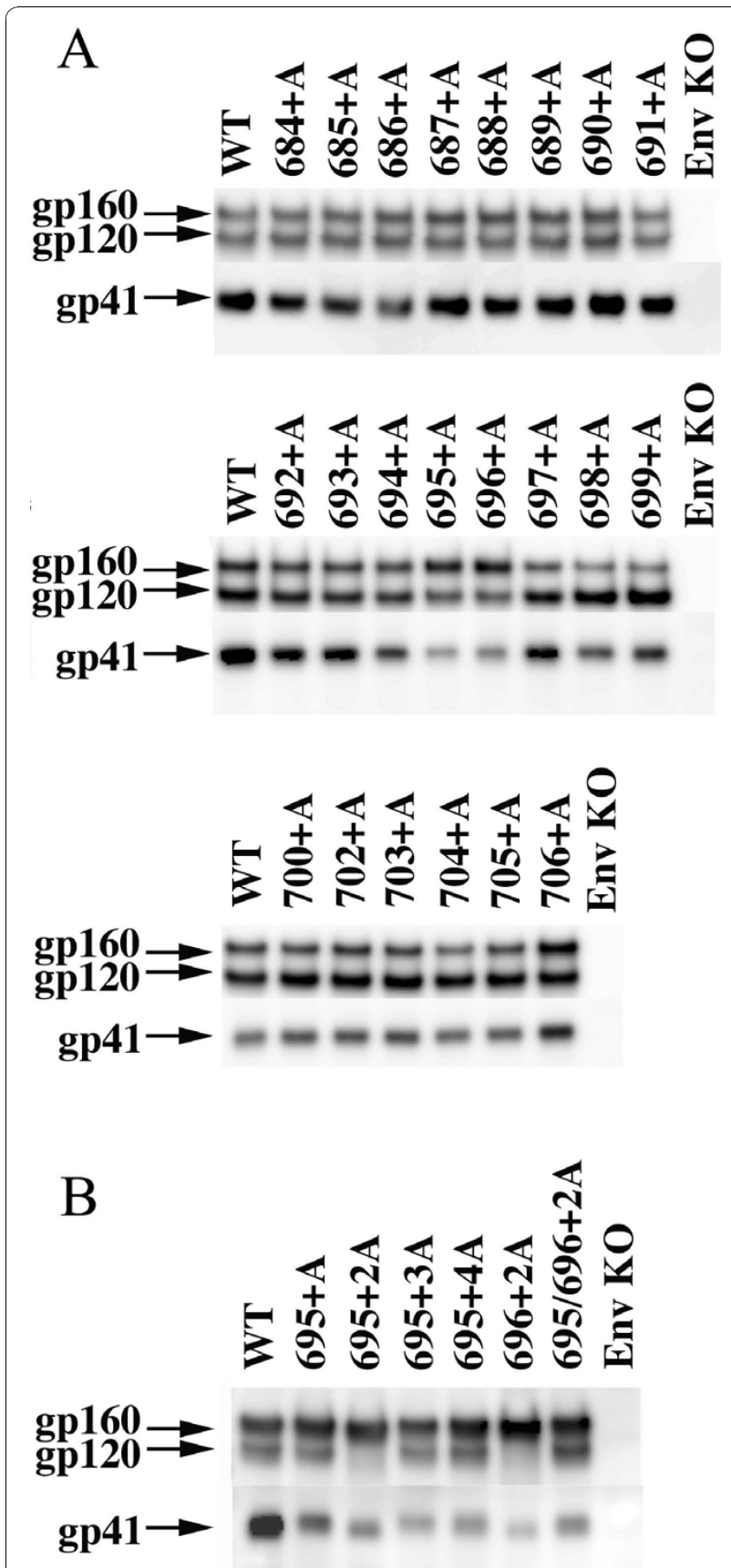

Figure 4 The immunoblotting analysis of wild type (WT) and Ala-inserted mutant Env. The envelope proteins expressed in COS-7 cells transfected with the Env expression vector were detected with anti-gp120 antibody (for gp160 and gp120) or with anti-gp41 antibody. The results of single- and multiple-Ala-insertion mutants are shown in $(A)$ and $(B)$, respectively.

transport (Figure 5) of Env. Since the processing of gp160 is dependent on the proper transport of the proteins to the Golgi apparatus, it seemed that the observed defect in processing might be due to a transport defect. However, we cannot rule out the possibility of a potential allosteric structural alteration of the Env by mutation in the MSD as a cause for the inefficient processing. Indeed our recent data suggested that the mutations in the gp41 MSD exert allosteric conformational changes of the ectodomain of HIV-1 Env [22].

Mutation at the cleavage site of gp160 eliminates HIV1 Env fusogenicity [7]. Thus, the defective membrane fusion of our alanine insertion mutants seemed to be derived from improper processing of gp160. However, there are other factors contributing to the defective fusion. Many studies have shown that mutations in the gp41 MSD affect membrane fusion efficiency $[18,23,24,29]$. In the context of $695+2 \mathrm{~A}$ mutant, the substitution of hydrophilic arginine residue with non-polar residues (alanine or isoleucine) rescues the defective processing (Additional file 3); however, this could not resolve the defective fusion (Additional file 4). These data suggest that gp41 MSD has a role(s) in the membrane fusion process itself. To reveal the exact mechanism, further studies are required.

It has been reported that MSD length is crucial for the trafficking of membrane proteins [31]. In HIV-1 Env, length of the MSD alone does not seem to be a primary determinant for trafficking. However, our data show that critical information lies in the local structure of the transmembrane $\alpha$-helix of gp41. It is possible that the alteration of structural features in the MSD region can be sensed by host factor(s) involved in the protein quality control system. This detection could be through the MSD region itself. In a yeast system, some proteins involved in the vesicular transport in ER-Golgi where target recognition was achieved via the MSD region have been reported [32,33]. Since the distribution of our Tac-gp41 chimera was heavily affected by the replacement of the MSD region alone (Figure 8), it may support such a hypothesis. Such a hypothetical factor may recognize wild type gp41 MSD via the GXXXG motif facing outward in relationship to the MSD bundle, if the gp41 MSDs interact with each other through arginine residues as suggested recently [30].

Notably, our alanine insertion mutation altered the relative positioning of the GXXXG motif and arginine residue within the gp41 MSD. Both are major interaction motifs between transmembrane $\alpha$-helices [34,35]. Although recent electron cryomicroscopic data [36-38] did not provide a spatial arrangement of the gp41 MSD portions, it is possible that there are interactions between the gp41 MSDs during the biosynthesis of the HIV-1 Env. Our alanine insertion may disrupt the interaction among MSDs. This disturbance of interhelical interactions may result in altered intracellular transport. The failure to reproduce differences in intracellular distribution between the wild type and $695+2 \mathrm{~A}$ MSD, in the context of Tac (Figure 8, B-I), may arise from the 


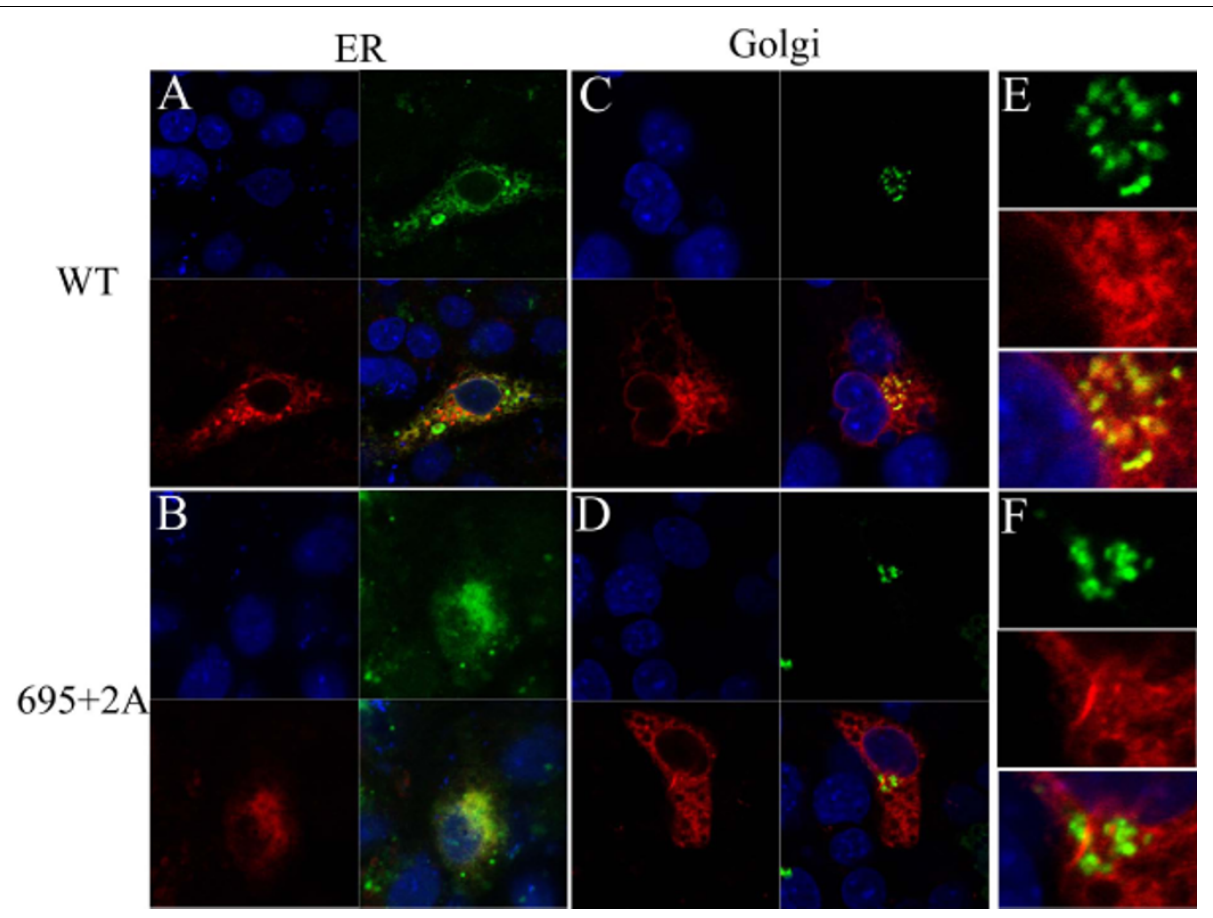

Figure 5 The transport defect of alanine insertion mutant Env. Endoplasmic reticulum (ER) ( $A$ and $B$ ) and Golgi (C to F) regions were visualized by fluorescence protein-conjugated ER or Golgi marker proteins (shown in green). FLAG tagged WT (A, C and E) and 695+2A Env

( $B, D$ and F) were stained by anti-FLAG antibody and Alexa Fluor (shown in red). The close-up of the Golgi area was shown in E and F. Nuclei of cells were stained with Hoechst 33258 (shown in blue).

difference in the oligomeric status between HIV-1 Env (trimer) and Tac (monomer). Our data suggest that mutant Env still forms a trimer (Additional file 2).

Our data clearly demonstrate that the MSD of gp41 has important functions in the biosynthesis of HIV-1 Env, apart from the simple anchoring and modulation of fusion efficiency. The exact regulation mechanism of intracellular distribution of HIV-1 Env by the MSD portion is not known; however, it could be of great importance to determine whether there are any cellular factors that specifically recognize the MSD region of HIV-1 Env.

\section{Conclusions}

We have shown that the secondary structure of the synthetic peptide of gp41 MSD is an $\alpha$-helix. Based on this information, we performed a scanning alanine insertion mutagenesis which showed that alteration of the topological relationship between conserved GXXXG motif and the arginine residue resulted in non-functional Env. The mutant Env manifested a reduced fusion activity and impaired the processing of gp160 into gp120 and gp41. Furthermore, the intracellular transport of mutant Env was affected in the endoplasmic reticulum and Golgi areas. Our data suggested that the specific $\alpha$-helical structural feature of gp41 MSD controls the biosynthesis of HIV-1 Env.

\section{Methods}

Synthesis of MSD peptides and its circular dichroism analysis

The sequence of the peptide used is KKWYIKIFIMIVGGLVGLRIVFAVLSIVNRKK, which corresponds to the consensus sequence of predicted MSD of clade B HIV-1. The sequence of the MSD of the clade B molecular clone, HXB2, used in this study differs by one amino acid from this sequence (indicated by the underline, HXB2 has $\mathrm{L}$ instead of I at this position). Two lysine residues were introduced at the $\mathrm{N}$ - and $\mathrm{C}$-termini to make the peptide more hydrophilic. The $\mathrm{CD}$ spectra were measured at $25^{\circ} \mathrm{C}$ with Aviv Model 215 (Aviv biomedical Inc, Lakewood, NJ) in $15 \mathrm{mM}$ DPC (n-dodecyl pyridinium chloride), $20 \mathrm{mM} \mathrm{NaPi}, 150 \mathrm{mM} \mathrm{NaCl}$. The concentration of the peptide was $10 \mu \mathrm{M}$. Eight spectra were averaged after subtracting for a DPC reference sample.

\section{Generation of the MSD mutants}

QuikChange Site-Directed Mutagenesis kit (Stratagene, La Jolla, CA) generated the mutants used in this study. The plasmid, pGEM7zNB, which contains the 1.2-kb NheIBamHI fragment covering the env portion of HXB2RU3 $\triangle \mathrm{N}$, was used as a template as described previously [18]. To facilitate the mutagenesis, silent restriction 

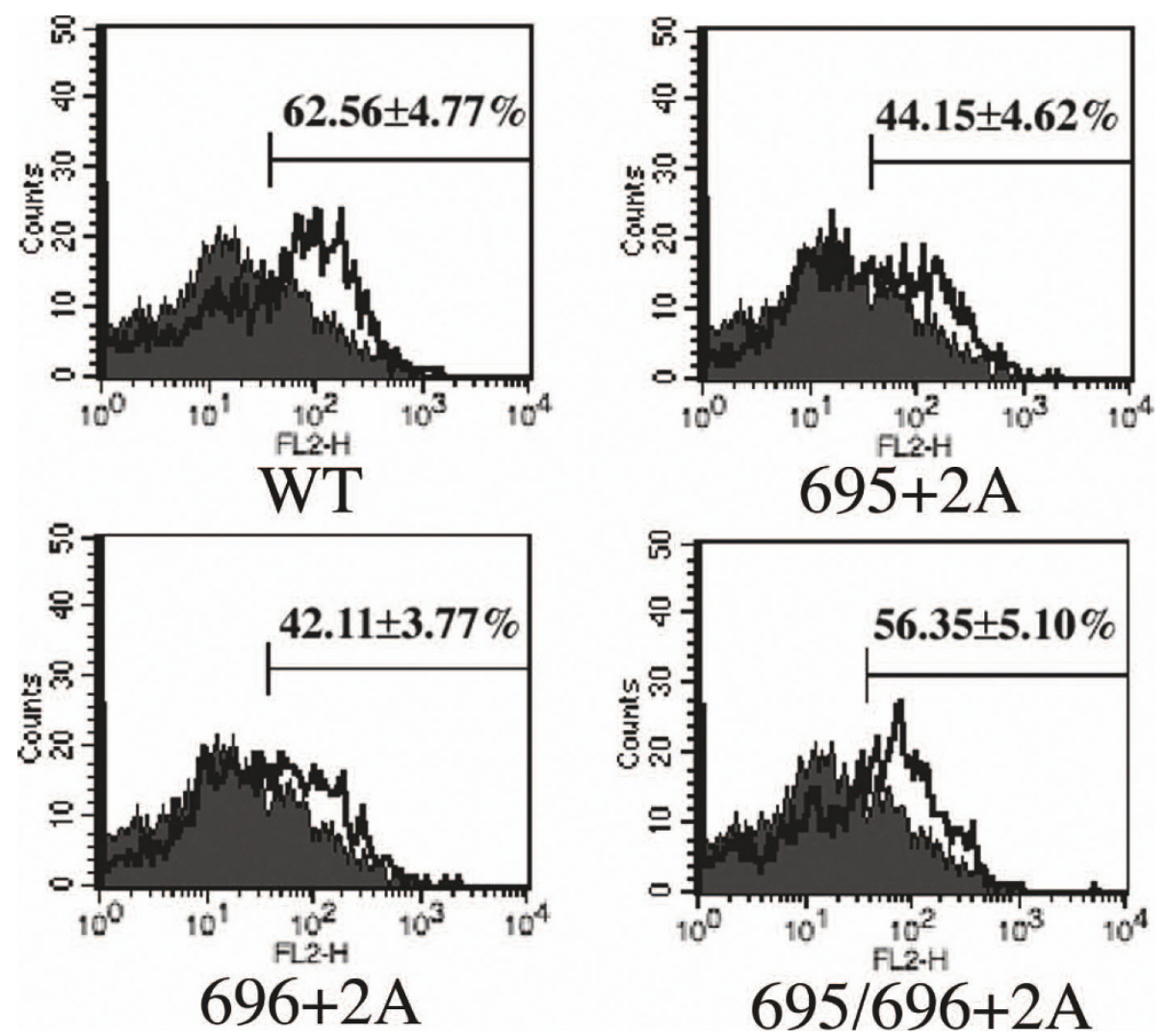

Figure 6 Surface expression level of Env. The cell surface expression level of envelope proteins for WT and Ala-insertion mutants on transfected COS-7 cells was determined by flow cytometry using anti-gp120 antibody.

enzyme sites for HindIII, SpeI, and BsiwI were generated near the MSD coding region. The complementary oligonucleotide pairs containing an inserted codon, GCC, for the alanine residue were cloned by using the HindIII, SpeI, and BsiwI sites. Multiple Ala-insertion mutants were made

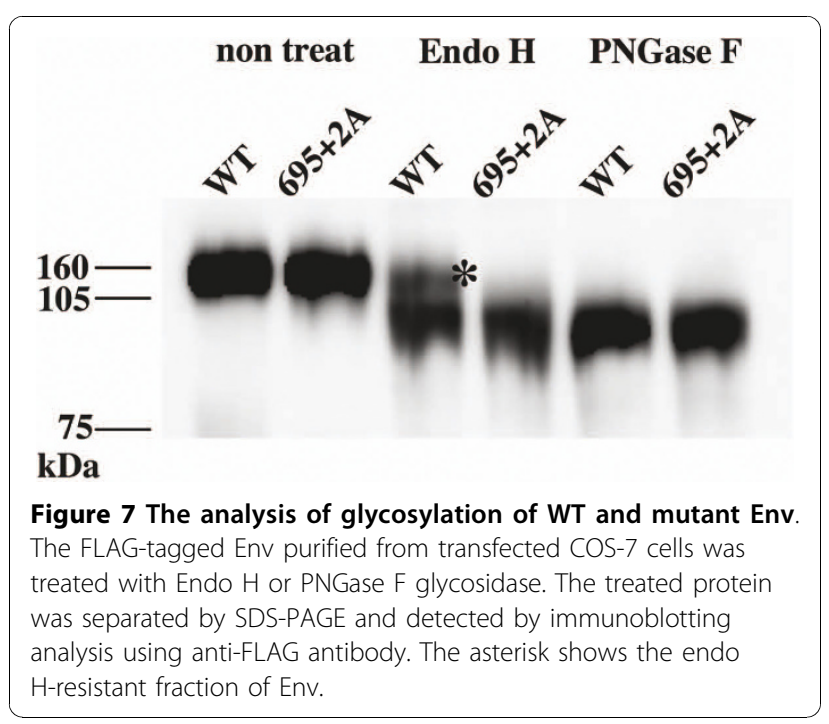

based on the single-insertion mutants. The complementary oligonucleotide pairs used were: 695+A, GGAGGCTTGG TAGGTGCTTT AAGAATAGTT TTT/AAAAACTATT CTTAAAGCAC CTACCAAGCC TCC, 696+A, GGCTTGGTAGGTTTAGCTAGAATAGTTTTTGCT/AGCAAAAACTATTCTAGCTAAAC CTACCAAGCC,695+2A,GAGGCTTGGTAGGTGCTG CCTTAAGAATAGTTTTTGC/GCAAAAACTATTCTTAAGGCAGCACCTACCAAGCCTC,695+3A, GTAG GAGGCTTGGTAGGTGCGGCCGCATTAAGAATAGTTTTTGCTGTACGTACAGCAAAAACTATTCTTAATGCGGCCGCACCTACCAAGCCTCCTAC, 695+4A, GGAGGCTTGGTAGGTGCGGCCGCAGCCTTAAGAATAGTTT TTGCTGTAC/GTACAGCAAAAACTATTCTTAAGGCTGCGGCCGCACCTACCAAGCCT CC,696+2A, GCTTGGTAGGTTTAGCTGCCAGAATAGTTTTTGCTG/CAGCAAAAACTATTCTGGCAG CTAAACCTACCAAGC,695/696+2A, GAGGCTTGGTAGGTGCTGCCTTAGCTGCCAGAATAGTTTTT GCTG/CAGCAAAAACTATTCTGGCAGCTAAGGCAG CACCTACCAAGCCTC. The NheI-BamHI fragment of pGEM7zNB containing the expected mutations was cloned back to pElucEnv [18] or pElucEnv-3FLAG Env (see below) expression vectors. 


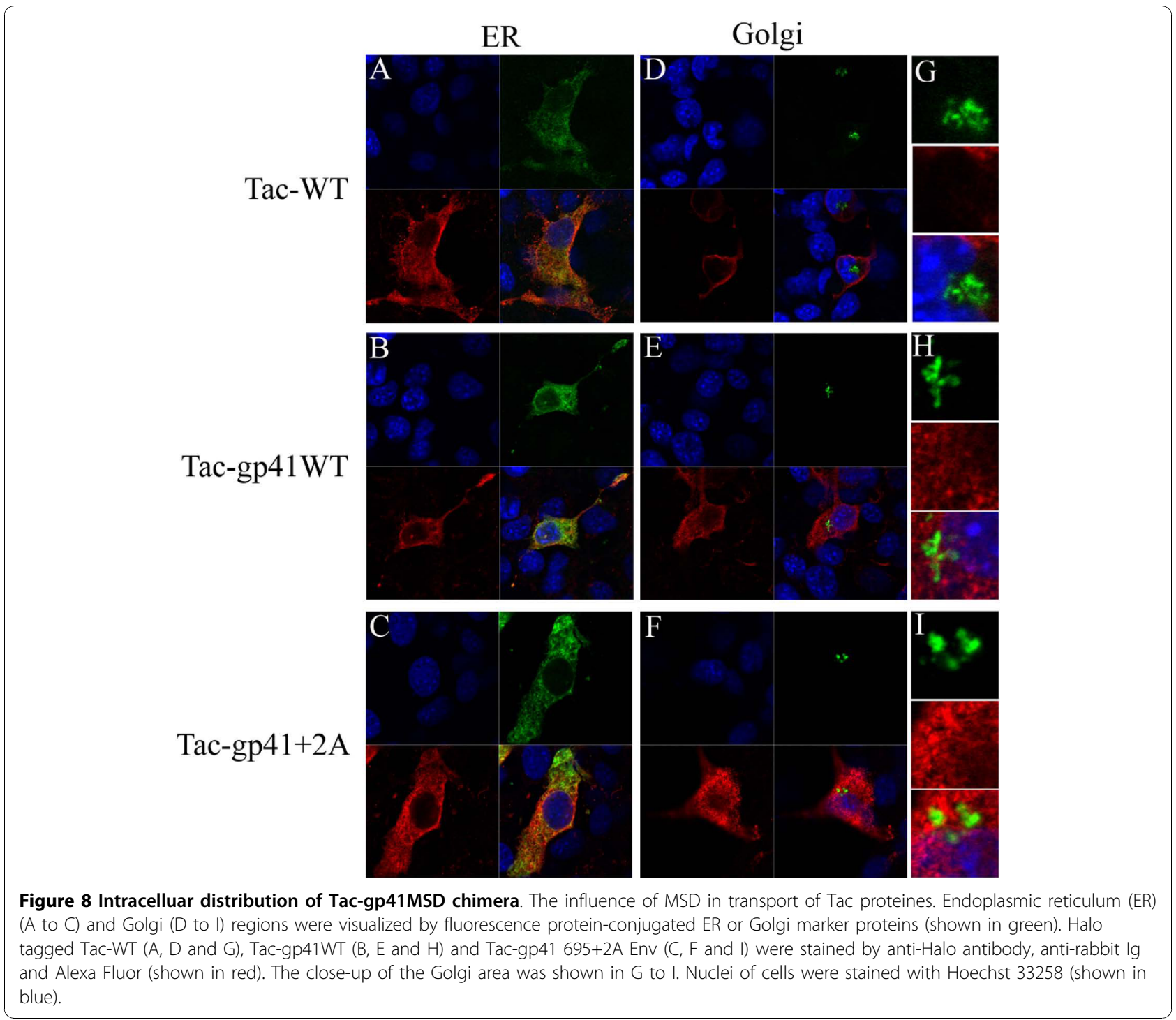

The synthetic codon-optimized gene corresponding to the Tac protein, $\alpha$-chain of Interleukin-2 receptor, with the gp41 MSD was custom synthesized (GenScript, Piscataway, NJ). The derivatives of this construct, whose MSD portion was replaced with those of wild type or mutant gp 41 or intact Tac, were generated by mutagenesis using PCR. These genes were cloned downstream of the CMV promoter to generate the Tac-derivative expression vectors.

\section{Addition of the $3 \times$ FLAG tag at the C-terminus of the Env}

A $3 \times$ FLAG tag was added to the C-terminus of gp 41 by inserting oligonucleotides corresponding to the $3 \times$ FLAG tag sequence derived from the vector p3xFLAG$\mathrm{CMV}^{\mathrm{m}}-7.1$ (Sigma, St. Louis, MO). Following this insertion, the amino acid sequence after the C-terminus of gp41 reads as RSARDYKDHDGDYKDHDIDYKDDDDK. The expression vector of FLAG-tagged Env was called pElucEnv-3FLAG Env.

\section{Cells and antibodies}

COS-7 cells, 293 cells, and 293-CD4 cells [18] were grown in Dulbecco's modified Eagle's medium (Sigma, St. Louis, MO) supplemented with $10 \%$ fetal bovine serum (HyClone Laboratories, Logan, UT) and penicillin-streptomycin (Invitrogen, Carlsbad, CA). Cells were kept under $5 \% \mathrm{CO}_{2}$ in a humidified incubator. Antigp120 polyclonal antibody was obtained from Fitzgerald Industries International, Inc. (Concord, MA). The hybridoma 902 and Chessie 8 were obtained from Bruce Chesebro and George Lewis, respectively through the AIDS Research and Reference Reagent Program, Division of AIDS, National Institute of Allergy and Infectious 
Diseases, National Institutes of Health, USA [39-41]. Anti-FLAG M2 and BioM2 were purchased from Sigma (St Louis, MO).

\section{Cell-cell fusion assay}

Cell-cell fusion assays, using T7 RNA polymerase (T7 RNA pol) transfer, were performed as described previously [18]. Briefly, 293-CD4 cells that constitutively express CD4 were transfected with pTM3hRL harboring the T7 promoter-driven renilla luciferase gene by FuGene 6 (Roche Applied Science, Mannheim, Germany), and were co-cultured with COS-7 cells that had been transfected with pCMMPT7iresGFP, a T7 RNA polymerase expression vector, and pElucEnv containing HIV-1 Env and firefly luciferase genes by FuGene 6 . After 12 hours of co-culture, the renilla and firefly luciferase activities were measured using the Dual-Glo luciferase assay system (Promega, Madison, WI). The fusion activity, represented by renilla luciferase activity, was normalized by firefly luciferase activity to obtain transfection efficiency [18]. The polyclonal anti-halo antibody was obtained from Promega (Promega, Madison, WI).

\section{Immunoblotting analysis}

$5 \times 10^{4}$ COS-7 cells were transfected with pElucEnv by FuGene 6 in a 24-well culture plate. Forty-eight hours after transfection, the cells were lysed with radioimmunoprecipitation assay lysis buffer $(0.05 \mathrm{M}$ TrisCl, 0.15 $\mathrm{M} \mathrm{NaCl}, 1 \%$ Triton $\mathrm{X}-100,0.1 \%$ sodium dodecyl sulfate, and $1 \%$ sodium deoxycholate). Cell lysates were electrophoresed (5-20\% Pantera Gel, DRC, Tokyo, Japan) and transferred to a polyvinylidene fluoride membrane (Pall, East Hills, NY). The blot was probed with anti-gp120 antibody (Fitzgerald, Concord, MA), with the monoclonal anti-gp41 antibody (Chessie 8), or with anti-FLAG M2 antibody. A biotinylated anti-species-specific immunoglobulin (GE Healthcare BioSciences AB, Uppsala, Sweden) was used as the secondary antibody. The blot was further treated with a streptavidin-horseradish peroxidase conjugate (GE Healthcare Bio-Sciences AB) and Lumi-Light ${ }^{\text {plus }}$ (Roche, Indianapolis, IN). Images were obtained with LAS3000 (Fujifilm, Tokyo, Japan).

\section{Immunofluorescence assay}

Immunofluorescence assays were used to determine the intracellular distribution of the envelope proteins. For this purpose, we generated a modified envelope expression vector called pElucEnvdeltaGFP; this is the derivative of the previously described pElucEnv [18] and it has the deletion of the EGFP portion. COS-7 cells transfected with pElucEnv WT or $695+2 \mathrm{~A}$ in the delta GFP backbone vector and ER-DsRed2 or GolgiYPF (Clontech) or pER-mAG1 (MBL, Nagoya, Japan) plasmid by FuGene 6 (Roche) were treated with PBS including $4 \%$ of PFA for $5 \mathrm{~min}$ at $48 \mathrm{hr}$ posttransfection. Cells were permeabilized by PBS including, $0.05 \%$ of saponin and $0.2 \%$ of BSA, for $30 \mathrm{~min}$ and then stained with $20 \mu \mathrm{g} / \mathrm{ml}$ of bio-M2 (Sigma) antibody and $10 \mu \mathrm{g} / \mathrm{ml}$ of streptavidin conjugated Alexa fluor 488 or 555 (Invitrogen). In the case of Halo-tagged proteins, polyclonal anti-Halo antibodies were used as primary antibodies. The distributions of fluorescence in cells were visualized using a Zeiss LSM 510 meta confocal microscope.

\section{Flow cytometric analysis}

Flow cytometric analysis was performed as described previously [18]. Briefly, COS-7 cells were transfected with pElucEnv by FuGene 6 on a six-well plate. Fortyeight hours aftertransfection, the cells were stained with anti-gp120 monoclonal antibody 902, biotinXX antimouse IgG (Invitrogen) and streptavidin-Alexa 555 in PBS including 10\% FBS. Cells were fixed with 1\% paraformaldehyde in PBS and analyzed by FACS Calibur (BD Biosciences).

\section{Glycosidase assay}

COS-7 cells transfected with pElucEnv-3FLAG by FuGene 6 on the six-well plate were lysed with radioimmunoprecipitation assay lysis buffer including Complete protease inhibitor (Roche). Env-3FLAG was purified from cell lysates by immunoprecipitation using M2 agarose (Sigma) and eluted with 3XFLAG peptide (Sigma). Purified Env-3FLAG was treated with Endo $\mathrm{H}$ or PNGase $F$ (Roche). For digestion by Endo H, Env3FLAG was boiled and digested with 0.005 unit Endo $\mathrm{H}$ at $37^{\circ} \mathrm{C}$ for $12 \mathrm{hr}$ in Endo $\mathrm{H}$ digestion buffer $[50 \mathrm{mM}$ phosphate buffer (pH 5.8), $50 \mathrm{mM} \mathrm{NaCl}, 0.1 \mathrm{M}$ 2-mercaptoethanol (2-ME), 0.01\% SDS]. Env-3FLAG was boiled in PBS including 0.1 M 2-ME and 0.1\% SDS and digested by 1 unit PNGase $\mathrm{F}$ at $37^{\circ} \mathrm{C}$ for $12 \mathrm{hr}$ in PNGase $\mathrm{F}$ digestion buffer (74 mM TrisCl, pH 8.0; $0.74 \%$ NP-40; 0.37 M 2-ME, 0.37\% SDS). Env-3FLAG treated with glycosidase was resolved by SDS-polyacrylamide gel electrophoresis (10\% polyacrylamide gel; DRC) and detected by immunoblotting analysis using antiFLAG M2.

\section{In vitro furin cleavage of Env}

Env-3FLAG with the $695+2 \mathrm{~A}$ mutation was purified from COS-7 cell lysates by immunoprecipitation as described above and treated with 0.7 units of furin (Alexis, Lausen, Switzerland) at $30^{\circ} \mathrm{C}$ for $12 \mathrm{hr}$ in furindigestion buffer (100 mM Hepes, $\mathrm{pH} 7.5 ; 1 \mathrm{mM} \mathrm{CaCl}_{2}$; $0.5 \%$ Triton $\mathrm{X}-100])$. Env-3FLAG, treated with furin, was detected by immunoblotting analysis using antiFLAG M2 as described above. 


\section{The cross linking analysis of Env}

At $48 \mathrm{hr}$ postransfection, 293T cells transfected with FLAG tagged WT or $695+2$ A Env expression vectors were treated with $1 \mathrm{mM}$ DSS for $20 \mathrm{~min}$ at room temperature in PBS (pH 8.0). Cells were incubated with 20 $\mathrm{mM}$ Tris- $\mathrm{Cl}$ for $15 \mathrm{~min}$ at room temperature to stop the reaction and then were lysed in buffer $A(10 \mathrm{mM}$ HEPES, $1.5 \mathrm{mM} \mathrm{MgCl}$, $10 \mathrm{mM} \mathrm{KCl}, 0.5 \mathrm{mM}$ DTT, $0.05 \%$ Igepal pH 7.9). Env proteins in cellular lysate were detected by immunoblotting analysis using antiFLAG antibody (see above).

\section{Additional material}

\section{Additional file 1: Supplemental Figure 1- In vitro digestion of} mutant Env with recombinant Furin. The wild type (WT) and mutant $(695+2 A)$ Env were prepared from transfected COS-7 cells and subjected to digestion with recombinant Furin (rFurin) as described in the Methods section. Mock indicates the result for the cell lysates prepared from mock transfected cells.

\section{Additional file 2: Suplemental Figure 2- Cross linking analysis of the} $695+2 A$ Env. The trimerization of gp160 was examined by chemical cross linking. The cells transfected with Env expression vectors for wild type (WT) and mutant $(695+2 A)$ were treated with the chemical cross linker. The cell lysates were probed with the anti-FLAG antibody. The single asterisk and the double asterisk indicate the bands for trimer and monomer of mutant gp160, respectively. Marker: HiMark Pre-Stained High Molecular Weight Protein Standard (Invitrogen), Mock: mock transfection.

Additional file 3: Suplemental Figure 3A - Immunoblotting analysis of the Arg-substitution mutants in the context of $695+2 \mathrm{~A}$. The degree of processing of gp160 was examined by immunoblotting the cell lysates prepared from COS-7 cells transfected with respective Env expression vectors. The Arg residue in the context of $695+2 \mathrm{~A}$ was substituted with the indicated amino acid residue by the site directed mutagenesis (columns under 2A). One letter abbreviation for an amino acid residue is used. Mock: mock transfection, WT: wild type MSD.

Additional file 4: Suplemental Figure 3B - Fusion activities of Argsubstitution mutants in the context of $695+2 \mathrm{~A}$. The fusion activities of the mutant shown in additional file $3 \mathrm{~A}$ were examined by a syncytia formation assay in 293CD4 cells. Fusion activity of the WT and MSD mutants was expressed using a fusion index (fusion index $=2 x+y_{1}$ where $x$ is the number of multinucleated cells [number of nuclei $\geq 5$ in five visual fields] and $y$ is the number of multinucleated cells [number of nuclei $<5$ in five visual fields]) as described previously [18].

\section{List of abbreviations}

MSD: membrane-spanning domain; CD: circular dichroism; ER: endoplasmic reticulum; WT: wild type.

\section{Acknowledgements}

This study was supported by a contract grant from the Ministry of Education, Culture, Sports, Science and Technology of Japan for the Program of Founding Research Centers for Emerging and Reemerging Infectious Diseases and a grant from the USNIH to DME (GM073857). We thank Dr. Kunito Yoshiike for his critical reading of the manuscript. We thank A. M. Menting, an editorial consultant, for help in the preparation of the manuscript.

\section{Author details}

'Laboratory of Virology and Pathogenesis, AIDS Research Center, National Institute of Infectious Diseases, 1-23-1 Toyama, Shinjuku, Tokyo, Japan.

${ }^{2}$ Department of Molecular Biophysics and Biochemistry, Yale University, Box
208114, New Haven, CT 06520-8114, USA. ${ }^{3}$ China-Japan Joint Laboratory of Structural Virology and Immunology, Institute of Biophysics, Chinese Academy of Sciences, 15 Datun Road, Beijing, 100101 PR China. ${ }^{4}$ Division of Infectious Diseases, Advanced Clinical Research Center, University of Tokyo, 4-6-1 Shirokanedai, Minato-ku, Tokyo, Japan. ${ }^{5}$ Research Center for Asian Infectious Diseases, Institute of Medical Science, University of Tokyo, 4-6-1 Shirokanedai, Minato-ku, Tokyo, Japan. ${ }^{6}$ Current Address: Department of Pediatrics, Emory University School of Medicine, 2015 uppergate Dr. Atlanta, GA 30322, USA.

\section{Authors' contributions}

KM, ARC, YL and NK performed most of the experimental work. KM, YL and $\mathbf{N K}$ did the cell biological analyses of mutant Envs. ARC analysed the synthetic peptide for its biophysical properties. Al contributed to discussion. DME and $\mathbf{Z M}$ conceived the study and coordinated the experiments. All authors read and approved the final manuscript.

\section{Competing interests}

The authors declare that they have no competing interests.

Received: 14 July 2010 Accepted: 13 November 2010 Published: 13 November 2010

\section{References}

1. Weiss CD: HIV-1 gp41: mediator of fusion and target for inhibition. AIDS Rev 2003, 5:214-221.

2. Eckert DM, Kim PS: Mechanisms of viral membrane fusion and its inhibition. Annu Rev Biochem 2001, 70:777-810.

3. Freed EO, Martin MA: The role of human immunodeficiency virus type 1 envelope glycoproteins in virus infection. J Biol Chem 1995, 270:23883-23886.

4. Gu M, Rappaport J, Leppla SH: Furin is important but not essential for the proteolytic maturation of gp160 of HIV-1. FEBS Lett 1995, 365:95-97.

5. Moulard M, Decroly E: Maturation of HIV envelope glycoprotein precursors by cellular endoproteases. Biochim Biophys Acta 2000 1469:121-132.

6. Ohnishi Y, Shioda T, Nakayama K, Iwata S, Gotoh B, Hamaguchi M, Nagai Y: A furin-defective cell line is able to process correctly the gp160 of human immunodeficiency virus type 1. J Virol 1994, 68:4075-4079.

7. McCune JM, Rabin LB, Feinberg MB, Lieberman M, Kosek JC, Reyes GR, Weissman IL: Endoproteolytic cleavage of gp160 is required for the activation of human immunodeficiency virus. Cell 1988, 53:55-67.

8. Kantanen ML, Leinikki P, Kuismanen E: Endoproteolytic cleavage of HIV-1 gp160 envelope precursor occurs after exit from the trans-Golgi network (TGN). Arch Virol 1995, 140:1441-1449.

9. Gabuzda D, Olshevsky U, Bertani P, Haseltine WA, Sodroski J: Identification of membrane anchorage domains of the HIV-1 gp160 envelope glycoprotein precursor. J Acquir Immune Defic Syndr 1991, 4:34-40.

10. Dimmock NJ: The complex antigenicity of a small external region of the C-terminal tail of the HIV-1 gp41 envelope protein: a lesson in epitope analysis. Rev Med Virol 2005, 15:365-381.

11. Lu L, Zhu Y, Huang J, Chen X, Yang H, Jiang S, Chen YH: Surface exposure of the HIV-1 env cytoplasmic tail LLP2 domain during the membrane fusion process: interaction with gp41 fusion core. J Biol Chem 2008, 283:16723-16731.

12. Haffar OK, Dowbenko DJ, Berman PW: Topogenic analysis of the human immunodeficiency virus type 1 envelope glycoprotein, gp 160, in microsomal membranes. J Cell Biol 1988, 107:1677-1687.

13. Helseth E, Olshevsky U, Gabuzda D, Ardman B, Haseltine W, Sodroski Changes in the transmembrane region of the human immunodeficiency virus type $1 \mathrm{gp} 41$ envelope glycoprotein affect membrane fusion. J Virol 1990, 64:6314-6318.

14. Yue L, Shang L, Hunter E: Truncation of the membrane-spanning domain of human immunodeficiency virus type 1 envelope glycoprotein defines elements required for fusion, incorporation, and infectivity. J Virol 2009, 83:11588-11598

15. West JT, Johnston PB, Dubay SR, Hunter E: Mutations within the putative membrane-spanning domain of the simian immunodeficiency virus transmembrane glycoprotein define the minimal requirements for fusion, incorporation, and infectivity. J Virol 2001, 75:9601-9612. 
16. Senes A, Engel DE, DeGrado WF: Folding of helical membrane proteins: the role of polar, GxxxG-like and proline motifs. Curr Opin Struct Biol 2004, 14:465-479.

17. Wilk T, Pfeiffer T, Bukovsky A, Moldenhauer G, Bosch V: Glycoprotein incorporation and HIV-1 infectivity despite exchange of the gp160 membrane-spanning domain. Virology 1996, 218:269-274.

18. Miyauchi K, Komano J, Yokomaku Y, Sugiura W, Yamamoto N, Matsuda Z: Role of the specific amino acid sequence of the membrane-spanning domain of human immunodeficiency virus type 1 in membrane fusion. Virol 2005, 79:4720-4729.

19. Deml L, Kratochwil G, Osterrieder N, Knuchel R, Wolf H, Wagner R: Increased incorporation of chimeric human immunodeficiency virus type 1 gp120 proteins into Pr55gag virus-like particles by an Epstein-Barr virus gp220/350-derived transmembrane domain. Virology 1997 235:10-25

20. Salzwedel K, Johnston PB, Roberts SJ, Dubay JW, Hunter E: Expression and characterization of glycophospholipid-anchored human immunodeficiency virus type 1 envelope glycoproteins. J Virol 1993, 67:5279-5288.

21. Owens RJ, Burke C, Rose JK: Mutations in the membrane-spanning domain of the human immunodeficiency virus envelope glycoprotein that affect fusion activity. J Virol 1994, 68:570-574.

22. Kondo N, Miyauchi K, Meng F, Iwamoto A, Matsuda Z: Conformational changes of the HIV-1 envelope protein during membrane fusion were inhibited by the replacement of its membrane-spanning domain. J Biol Chem 2010, 285:14681-8.

23. Miyauchi K, Curran R, Matthews E, Komano J, Hoshino T, Engelman DM, Matsuda Z: Mutations of conserved glycine residues within the membrane-spanning domain of human immunodeficiency virus type 1 gp41 can inhibit membrane fusion and incorporation of Env onto virions. Jpn J Infect Dis 2006, 59:77-84

24. Shang $L$, Yue $L$, Hunter $E$ : Role of the membrane-spanning domain of human immunodeficiency virus type 1 envelope glycoprotein in cell-cell fusion and virus infection. J Virol 2008, 82:5417-5428.

25. Ratner L, Fisher A, Jagodzinski LL, Liou RS, Mitsuya H, Gallo RC, WongStaal F: Complete nucleotide sequences of functional clones of the virus associated with the acquired immunodeficiency syndrome, HTLV-III/LAV. Haematol Blood Transfus 1987, 31:404-406.

26. Andreassen $H$, Bohr H, Bohr J, Brunak S, Bugge T, Cotterill RM, Jacobsen C, Kusk P, Lautrup B, Petersen SB, et al: Analysis of the secondary structure of the human immunodeficiency virus (HIV) proteins p17, gp120, and gp41 by computer modeling based on neural network methods. J Acquir Immune Defic Syndr 1990, 3:615-622.

27. Pace CN, Scholtz JM: A helix propensity scale based on experimental studies of peptides and proteins. Biophys J 1998, 75:422-427.

28. Chakrabartty A, Schellman JA, Baldwin RL: Large differences in the helix propensities of alanine and glycine. Nature 1991, 351:586-588.

29. Welman $M$, Lemay $G$, Cohen EA: Role of envelope processing and gp41 membrane spanning domain in the formation of human immunodeficiency virus type 1 (HIV-1) fusion-competent envelope glycoprotein complex. Virus Res 2007, 124:103-112.

30. Kim JH, Hartley TL, Curran AR, Engelman DM: Molecular dynamics studies of the transmembrane domain of gp41 from HIV-1. Biochim Biophys Acta 2009, 1788:1804-1812.

31. Ronchi P, Colombo S, Francolini M, Borgese N: Transmembrane domaindependent partitioning of membrane proteins within the endoplasmic reticulum. J Cell Biol 2008, 181:105-118.

32. Sato $K$, Sato M, Nakano A: Rer1p, a retrieval receptor for ER membrane proteins, recognizes transmembrane domains in multiple modes. $\mathrm{Mol}$ Biol Cell 2003, 14:3605-3616.

33. Reggiori F, Black MW, Pelham HR: Polar transmembrane domains target proteins to the interior of the yeast vacuole. Mol Biol Cell 2000, 11:3737-3749.

34. Curran AR, Engelman DM: Sequence motifs, polar interactions and conformational changes in helical membrane proteins. Curr Opin Struct Biol 2003, 13:412-417

35. Cosson P, Lankford SP, Bonifacino JS, Klausner RD: Membrane protein association by potential intramembrane charge pairs. Nature 1991, 351:414-416.
36. Zhu P, Liu J, Bess J, Chertova E, Lifson JD, Grise H, Ofek GA, Taylor KA, Roux $\mathrm{KH}$ : Distribution and three-dimensional structure of AIDS virus envelope spikes. Nature 2006, 441:847-852

37. Liu J, Bartesaghi A, Borgnia MJ, Sapiro G, Subramaniam S: Molecular architecture of native HIV-1 gp120 trimers. Nature 2008, 455:109-113.

38. Zhu P, Winkler H, Chertova E, Taylor KA, Roux KH: Cryoelectron tomography of HIV-1 envelope spikes: further evidence for tripod-like legs. PLOS Pathog 2008, 4:e1000203.

39. Chesebro B, Wehrly K: Development of a sensitive quantitative foca assay for human immunodeficiency virus infectivity. J Virol 1988, 62:3779-3788

40. Pincus SH, Wehrly $\mathrm{K}$, Chesebro B: Treatment of HIV tissue culture infection with monoclonal antibody-ricin A chain conjugates. J Immunol 1989, 142:3070-3075

41. Abacioglu YH, Fouts TR, Laman JD, Claassen E, Pincus SH, Moore JP, Roby CA, Kamin-Lewis R, Lewis GK: Epitope mapping and topology of baculovirus-expressed HIV-1 gp160 determined with a panel of murine monoclonal antibodies. AIDS Res Hum Retroviruses 1994, 10:371-381.

doi:10.1186/1742-4690-7-95

Cite this article as: Miyauchi et al:: The membrane-spanning domain of gp41 plays a critical role in intracellular trafficking of the HIV envelope protein. Retrovirology 2010 7:95.

\section{Submit your next manuscript to BioMed Central and take full advantage of:}

- Convenient online submission

- Thorough peer review

- No space constraints or color figure charges

- Immediate publication on acceptance

- Inclusion in PubMed, CAS, Scopus and Google Scholar

- Research which is freely available for redistribution

Submit your manuscript a www biomedcentral com/submit
Biomed Central 